\title{
In situ Measurement of Diamond/SiC Interfacial Strength
}

\author{
Yuying Zhang ${ }^{1}$, Chun-Yen Hsu ${ }^{1}$, Yong Zhao ${ }^{1}$, Prashant Karandikar ${ }^{1,2}$ and Chaoying $\mathrm{Ni}^{1 *}$ \\ 1. Department of Materials Science and Engineering, University of Delaware, Newark, DE, USA. \\ 2. M cubed Technologies Inc., Newark, DE, USA. \\ * Corresponding author: cni@udel.edu
}

Reaction bonded (RB) diamond/SiC composites were prepared by pressureless liquid Si infiltration technique. Reaction bonding fabrication starts with preparing diamond/SiC porous preform and then infiltrates molten $\mathrm{Si}$ at a temperature of $\sim 1450{ }^{\circ} \mathrm{C}$ under a vacuum condition. The resulting products are fully dense with advanced thermal and mechanical properties which make them premier candidates for applications in defense, high energy laser mirror, semiconductor fabrication, machining and aerospace industries. Because of the high processing temperature, preform component selection and phase formation complexities, the $\mathrm{RB}$ diamond/SiC composite properties are a strong function of the resulting microstructures, especially the interfaces. Liu et al., [1] reported that the diamond particle size in the preform and composite porosity strongly affect the surface hardness, flexural strength, and fracture toughness. Tressler et al.[2] studied the crack propagation inside the $\mathrm{RB} \mathrm{SiC} / \mathrm{Si}$ composites and proposed two failure mechanisms which could affect the mechanical performance. Interfacial strength and intergranular failure are important parameters for understanding and evaluating the mechanical behavior of the composite, especially for composites with interfacial complexities such as those in RB composites. However, to the best of the authors' knowledge, there was limited investigation on the interfacial bonding strength of the RB diamond/SiC composites. In this work, we developed a home-made tensile stage for in-situ study of the interfacial strength of diamond/SiC.

The microstructure of RB diamond/SiC composite was studied by using a FIB/SEM (Zeiss Auriga 60) equipped with an X-ray energy-dispersive spectrometer (EDS, Oxford X-Max 80). Dog-bone specimens were prepared by using FIB with Nano-Patterning and Visualization Engine (NPVE). FT-S microforce sensing probes $\left(\mathrm{F}_{\max }=1000 \mu \mathrm{N}\right.$, FemtoTools) were used for capturing the force-time curve and a video was recorded (frame/100ms) for analyzing the elongation of the specimen.

As shown in Fig 1(a), the microstructure of $\mathrm{RB}$ diamond/SiC composite was collected by using a backscattered electron (BSE) detector at accelerating voltage of $10 \mathrm{kV}$. The sample contains diamond (D, black), SiC (grey), and Si (light grey), respectively, as confirmed by EDS maps of carbon and Si. Fig 1(b) shows a cross-section of diamond/SiC interface. EDS analysis performed for the square region suggests that the bright phase corresponds to an $\mathrm{Al}$ compound that is further confirmed to be $\mathrm{Al}_{4} \mathrm{C}_{3}$ by $\mathrm{TEM}$ electron diffraction.

As shown in Fig 2(a), the dog-bone sample with diamond/SiC interface is mounted between the force sensor (left) and a Si fixture (right). A zoom-in image (upright inset) of the square region suggests the existence of the $\mathrm{Al}_{4} \mathrm{C}_{3}$ in this interfacial region. During the tensile test, a brittle fracture was observed to occur at the interface (downright inset) with a fracture strength of $0.73 \mathrm{GPa}$ and a typical stress-strain curve is shown in Fig 2(b). Compared with the strength of diamond (0.89 2.42 GPa [3]) and $\mathrm{SiC}(1.19$ $\pm 0.53 \mathrm{GPa}[4])$ and the average interfacial strength $(0.75 \mathrm{GPa})$ in our other tests, the interphase $\mathrm{Al}_{4} \mathrm{C}_{3}$ appears to degrade the interfacial strength of diamond/SiC and may have detrimental effect on the composite properties. 
In addition to the three major phases of diamond, $\mathrm{SiC}$ and $\mathrm{Si}$ in $\mathrm{RB}$ diamond/SiC composite, small quantity of $\mathrm{Al}_{4} \mathrm{C}_{3}$ was observed near the interfacial region. During the tensile test, the fracture happened at the interfacial region with the interphase $\mathrm{Al}_{4} \mathrm{C}_{3}$ suggesting the interface with impurity interphases can be a weak link in the diamond/SiC composite and high quality raw materials may further promote the mechanical properties of this advanced composite [5].

\section{References:}

[1] YS Liu et al., J. Eur. Ceram. Soc. 34 (2014), p. 3489. doi:10.1016/j.jeurceramsoc.2014.05.042.

[2] R Tressler et al., in "Tailoring Multiphase and Composite Ceramics", (Springer Science \& Business Media).

[3] HD Espinosa et al., MRS Proceedings (2002), p. 256.

[4] KM Jackson et al., J. Microelectromechanical Syst. 14 (2005), p. 664.

[5] This work is supported by the II-VI Foundation. Instrumentation and staff supports from the W. M. Keck Center for Advanced Microscopy and Microanalysis at the University of Delaware are greatly appreciated
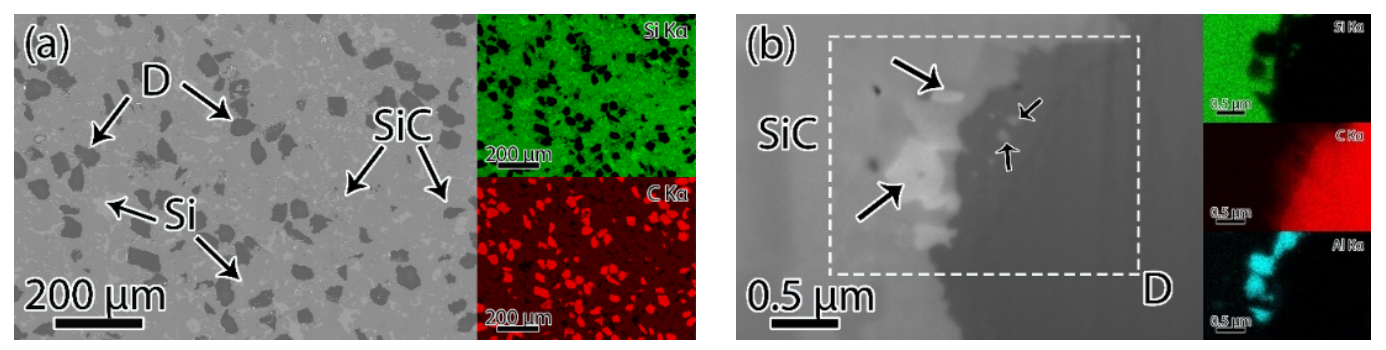

Figure 1. SEM images and EDS maps of RB diamond/SiC composite: (a) SEM image captured by BSE detector, (b) SEM image captured by in-lens detector
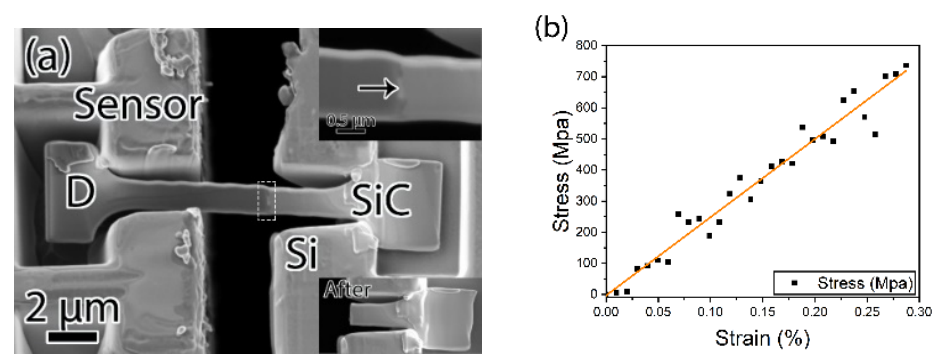

Figure 2. In-situ tensile test: (a) SEM image of sample before and after test, (b) stress-strain curve for diamond $/ \mathrm{SiC}$ interface 